\title{
Assessing the accuracy of ultrasound measurements of tracheal diameter: an in vitro experimental study
}

Ran Ye ${ }^{1+}$, Feifei $\mathrm{Cai}^{2 \dagger}$, Chengnan Guo ${ }^{3}$, Xiaocheng Zhang ${ }^{4}$, Dan Yan ${ }^{5}$, Chengshui Chen ${ }^{4,6^{*}}$ (D) and Bin Chen ${ }^{7 *}$

\begin{abstract}
Background: Recent studies indicate that ultrasound can detect changes in tracheal diameter during endotracheal tube (ETT) cuff inflation. We sought to assess the accuracy of ultrasound measurement of tracheal diameter, and to determine the relationship between tracheal wall pressure (TWP), cuff inflation volume (CIV), and the degree of tracheal deformation.
\end{abstract}

Methods: Our study comprised two parts: the first included 45 porcine tracheas, the second 41 porcine tracheas. Each trachea was intubated with a cuffed ETT, which was connected to an injector and the manometer via a threeway tap. The cuff was inflated and the cuff pressure recorded before and after intubation. The tracheal diameter was measured using ultrasound. This included three separate measurements: outer transverse diameter (OTD), internal transverse diameter (ITD), and anterior tracheal wall thicknesses (ATWT). A precision electronic Vernier caliper was also used to measure tracheal diameter. We calculated TWP and the percentage change of tracheal diameter. The Bland-Altman method, linear regression, and locally weighted regression (LOESS) were used to analyze the data.

Results: There were strong correlation and agreement for OTD $(r=0.97, P<0.001)$ and ITD $(r=0.90, P<0.001)$ as measured by ultrasound and by precision electronic Vernier caliper, but a poor correlation for ATWT $(r=0.58, P<$ 0.001). There was a strong correlation between the percentage change of OTD (OTD $\%, r=0.75, P<0.001)$ and CIV, the percentage change of ITD (ITD\%, $r=0.77, P<0.001)$ and CIV, TWP $(r=0.75, P<0.001)$ and CIV. And a strong correlation was also found between TWP and OTD\% $(r=0.84, P<0.001)$, TWP and ITD\% $(r=0.84, P<0.001)$.

Conclusions: Use of ultrasound to measure OTD and ITD is accurate, but is less accurate for ATWT. There is a close correlation between OTD\%, ITD\%, CIV and TWP.

Keywords: Ultrasound, Endotracheal tube, Tracheal diameter, Tracheal wall pressure, Cuff inflation volume

\footnotetext{
*Correspondence: ccswenz@163.com; doctorchbe@126.com

${ }^{\dagger}$ Ran Ye and Feifei Cai contributed equally to this work.

${ }^{4}$ Key Laboratory of Interventional Pulmonology of Zhejiang Province, The First Affiliated Hospital of Wenzhou Medical University, Wenzhou 325006, Zhejiang, China

${ }^{7}$ Department of Ultrasonography, The First Affiliated Hospital of Wenzhou Medical University, Wenzhou 325006, Zhejiang, China

Full list of author information is available at the end of the article
}

(C) The Author(s). 2021 Open Access This article is licensed under a Creative Commons Attribution 4.0 International License, which permits use, sharing, adaptation, distribution and reproduction in any medium or format, as long as you give appropriate credit to the original author(s) and the source, provide a link to the Creative Commons licence, and indicate if changes were made. The images or other third party material in this article are included in the article's Creative Commons licence, unless indicated otherwise in a credit line to the material. If material is not included in the article's Creative Commons licence and your intended use is not permitted by statutory regulation or exceeds the permitted use, you will need to obtain permission directly from the copyright holder. To view a copy of this licence, visit http://creativecommons.org/licenses/by/4.0/ The Creative Commons Public Domain Dedication waiver (http://creativecommons.org/publicdomain/zero/1.0/) applies to the data made available in this article, unless otherwise stated in a credit line to the data. 


\section{Background}

Airway ultrasound can provide detailed images of the upper airway, including the thyroid cartilage, vocal cords, and trachea $[1,2]$. Ultrasound has been shown to detect accurately the position [3-5] and depth of endotracheal tube (ETT) [6, 7] during intubation. The tracheal diameter measured by ultrasound is the basis of several studies. Certain studies $[8,9]$ indicated that ultrasound measurement of tracheal diameter is reliable. An animal study measured the tracheal diameter to assess tracheal collapse [10]. Clinical studies demonstrate that laryngeal ultrasonography can measure width difference of the air column before and after deflation of endotracheal tube cuff, which may be a predictor of post-extubation stridor $[11,12]$.

To the best of our knowledge, the accuracy and veracity of ultrasound measurements can depend on the experience of the sonographer. Stuntz [13] and Gottlieb [14] both observe that, when compared with the clinician, the professional sonographers obtain better airway ultrasound images and interpret images with greater accuracy and acuity. Chou [15] indicates that with proper training, clinicians can undertake airway ultrasonography and obtain accurate and reliable results. Julio [16] trained three second-year anesthesiology residents, showing tracheal internal transverse diameter measurements obtained by different operators were both reliable and precise.

Endotracheal intubation is often performed in patients with respiratory failure. It maintains airway patency by establishing artificial airways, and supports subsequent mechanical ventilation. The cuff is inflated with air to create a seal within the airway. This helps maintain positive pressure ventilation and prevents micro-aspiration of fluid secretion. Many studies recommend cuff pressure is monitored and kept between 20 and $30 \mathrm{cmH}_{2} \mathrm{O}$ [17]. Tracheal wall pressure (TWP) is the pressure that endotracheal cuff exerts on tracheal wall. Despite the high-volume, low-pressure cuff pressure is related to tracheal wall pressure, but it is not exactly the same. If tracheal wall pressure does not exceed the capillary pressure of tracheal mucosa, complications arising from intubation are reduced [18]. Ramsingh $e l$ at. showed that inflation of endotracheal tube cuff increases trachea diameter, which can be observed using ultrasound $[6,19]$. As tracheal wall pressure and tracheal deformation are caused by inflation of endotracheal tube cuff, a correlation is possible between tracheal wall pressure, cuff inflation volume (CIV), and the degree of tracheal deformation as determined by ultrasound.

In this study, our primary aim was to assess the accuracy of ultrasound measurements of the three tracheal diameters. Our second aim was to explore the relationship between tracheal wall pressure, cuff inflation volume, and the degree of tracheal deformation as measured by ultrasound.

\section{Methods \\ Materials}

All animal studies were conducted under the oversight of the Institutional Animal Care and Use Committee of Wenzhou Medical University (Wenzhou, China). In the present study, 45 porcine tracheas were obtained from animals sacrificed within $24 \mathrm{~h}$ in local abattoirs. No living animals were used in this study. Each tracheal specimen consisted of the upper larynx, trachea, and part of the right and left main stem bronchus.

An ultrasonography device (EZU-MT28-S1, HITA CHI, Japan, Tokyo) with a $5-13 \mathrm{~Hz}$ linear probe was used for ultrasonography. The balloon of ETT was connected to a $10 \mathrm{~mL}$ injector and a digital manometer (PLD.0201, BOOST, China) through a three-way tap. The range of the digital manometer is $0-35 \mathrm{kPa}$ and the accuracy $0.2 \%$.

As the anterior cervical tissue was not present in the porcine trachea, ultrasound images were affected by the presence of air. We prepared a thin-walled approximately $150 \mathrm{~mm}$ long water bladder by loading $100 \mathrm{~mL}$ water into a condom. The water bladder was placed perpendicular to the long axis of the trachea. The water bladder was light and soft, and was used as acoustic window to allow proper display of resulting tracheal images [20]. Tracheal structure remained mostly unchanged. Ultrasonography gel was applied between the tracheal surface and the water bladder.

The trachea was positioned so that it lay flat supported on a rigid bracket on the table. It was then intubated with an $8.0 \mathrm{~mm}$ oral/nasal tracheal tube (Covidien, USA, Mansfield). The outer diameter of the inflation cuff is $27 \mathrm{~mm}$. An ETT was placed at a depth of $18 \mathrm{~mm}$, based on the distance from the thyroid cartilage. Tape was used to secure the porcine trachea to the rigid bracket, ensuring a constant position during measurement. A transverse line was drawn on the trachea to mark the center of the cuff.

\section{Ultrasonographical features of the porcine trachea}

The porcine trachea was semicircular in the transverse plane, resembling an inverted $U$. The cartilage of the tracheal rings was hypoechoic; if calcification occurs, it may become hyperechoic. The outer edge of the trachea presented a hyperechoic strip with a clear smooth boundary. The inner surface of the trachea was linearly hyperechoic, and is known as the air-mucosal interface (A-M interface). The posterior part of the trachea was the reverberation artifact.

The outer transverse diameter (OTD) was defined as the distance between the hyperechoic regions on both 
sides of the tracheal edge. The internal transverse diameter (ITD) was defined as the distance between the A-M interfaces of both sides. The ITD showed a hypoechoic edge on the ultrasound. The anterior tracheal wall thickness (ATWT) was defined as the distance from the hyperechoic front wall to the A-M interface (Fig. 1).

\section{Pressure difference technique}

The TWP was estimated using the following formula [21]:

$$
\mathrm{TWP}=\mathrm{CP}_{\text {inserted }}-\mathrm{CP}_{\text {uninserted }}
$$

The uninserted cuff pressure $\left(\mathrm{CP}_{\text {uninserted }}\right)$ is a ETT cuff pressure measured after inflating with a set volume of air in vitro. The inserted cuff pressure $\left(\mathrm{CP}_{\text {inserted }}\right)$ is the pressure generated after the ETT intubated into the trachea and the cuff inflated with the same volume of air. In the same ETT, the pressure generated after every $1 \mathrm{~mL}$ increment of inflation with air was measured using the digital manometer.

\section{Study process}

A researcher injected air into the uninserted ETT cuff (with an incremental increase of $1 \mathrm{~mL}$ ) through a threeway tap while recording $\mathrm{CP}_{\text {uninserted }}$ and CIV. After the ETT was inserted into the porcine trachea, the same researcher repeated this procedure, recording $\mathrm{CP}_{\text {inserted }}$. A professional sonographer used a $5-13 \mathrm{~Hz}$ linear probe to acquire the transverse plane image of each CIV. Ultrasonography was performed directly above the marker line, with the probe perpendicular to the table. Measurements were taken until CIV reached $10 \mathrm{~mL}$. All measurements were repeated three times. A new ETT was used for each porcine trachea.
OTD, ITD, and ATWT were measured by the sonographer. The percentage change of OTD (OTD\%) was calculated using the following formula:

$$
\mathrm{OTD} \%=(\mathrm{OTD}-\mathrm{OTD} 0) / \mathrm{OTD} 0 \times 100
$$

The OTD was measured for each CIV. OTD0 was the OTD of the trachea when the CIV was $0 \mathrm{~mL}$. The percentage change of ITD (ITD\%) was calculated similarly. The percentage change of ATWT (ATWT\%) was calculated using the following formula:

$$
\text { ATWT\% }=(\text { ATWT0-ATWT }) / \text { ATWT0 } \times 100 .
$$

When $\mathrm{CP}_{\text {uninserted }}, \mathrm{CP}_{\text {inserted }}$ OTD, ITD, and ATWT of a given trachea were measured, a precision electronic Vernier caliper was used to measure the OTD along the marked line after removal of the ETT. A cross transverse incision was made through the trachea to measure the ITD and ATWT. Each diameter was assessed three times by a researcher.

\section{Statistical analysis}

Statistical analyses were conducted using MedCalc19.0.4 and SAS9.4. The distribution of continuous variables was analyzed using the Kolmogrov-Smirnov test. Normally distributed variables were summarized as mean and standard deviation. Non-normally distributed variables were presented as median and by the interquartile range. The Bland-Altman method and linear regression were used to assess the accuracy of and the agreement between measurements made using the precision electronic Vernier caliper and by ultrasound. Locally weighted regression (LOESS) was used to observe changes in TWP, CIV and the percentage change of tracheal diameter. Pearson correlation analysis was used to assess normally distributed variables and

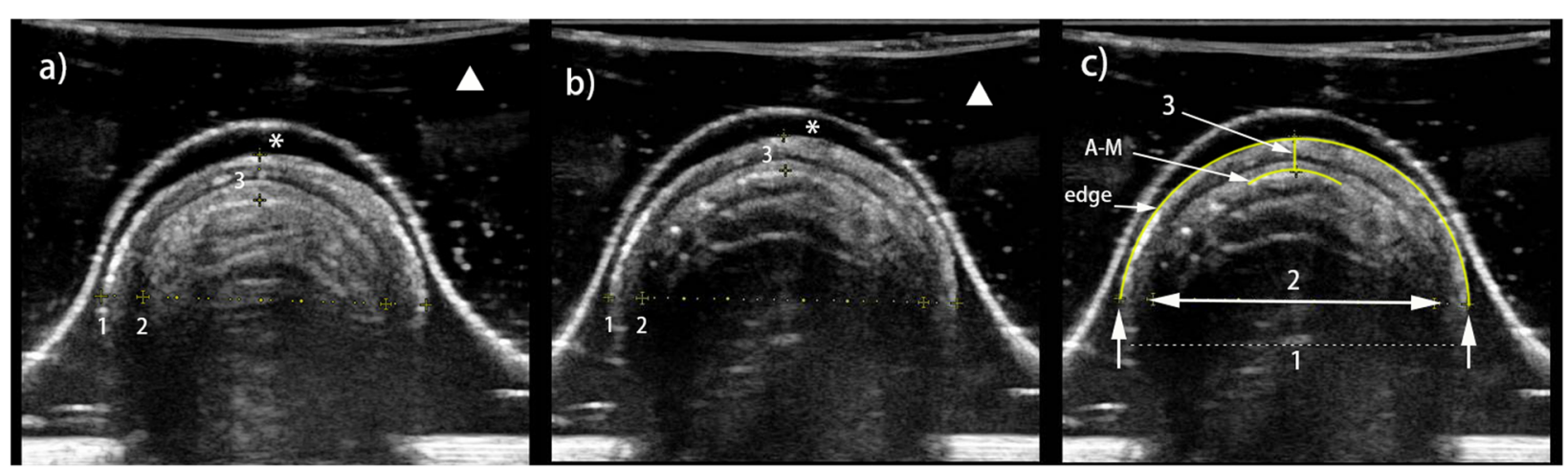

Fig. 1 The sonogram shows the porcine trachea in the transverse plane. Water bladder $(\mathbf{\Delta})$; ultrasonography gel $\left(^{*}\right)$; lines indicate outer transverse diameter (OTD) (1), internal transverse diameter (ITD) (2) and anterior tracheal wall thicknesses (ATWT) (3). a Cuff inflation volume is 0 $\mathrm{mL}$. b Cuff inflation volume is $10 \mathrm{~mL}$. c Tracheal diameter measurement. OTD (width between two white arrows; dotted line); ITD (two white arrowheads); ATWT (yellow vertical line); A-M interface (A-M); tracheal outer edge 
Spearman correlation analysis was used to assess nonnormally distributed variables. A $P$ value $<0.05$ was considered significant.

\section{Results}

Analysis of the agreement and accuracy in the measurement of the tracheal diameter using ultrasound The first part included 45 porcine tracheas. Sample size calculation and power analysis were performed (Supplementary Figure 1, 2 and 3). Table 1 shows the measurements of the tracheal diameter using both ultrasound and the precision electronic Vernier caliper.

Bland-Altman analysis and linear regression, indicated a strong correlation between precision electronic Vernier caliper and ultrasonography measurements of OTD $(n=$ $45, r=0.97, P<0.001)$. We noted a bias of $-0.19 \mathrm{~mm}$ with a precision of $0.08 \mathrm{~mm}$. The limit of agreement was $-1.19 / 0.80 \mathrm{~mm}$ (Fig. 2A). With respect to ITD, there was also a strong correlation between the methods $(n=$ $45, r=0.90, P<0.001)$. We observed a bias of $0.33 \mathrm{~mm}$ with a precision of $0.14 \mathrm{~mm}$. The limit of agreement was $-1.45 / 2.11 \mathrm{~mm}$ (Fig. 2B).

For ATWT, there was a poor correlation between the methods $(n=45$, Spearman's rank correlation coefficient: $0.58, P<0.001)$. We noted a bias of $0.23 \mathrm{~mm}$ with a precision of $0.07 \mathrm{~mm}$. The limit of agreement was -0.75 / $1.20 \mathrm{~mm}$ (Fig. 2C).

The relationship between the tracheal diameter, tracheal wall pressure, and cuff inflation volume

The second part included 41 porcine tracheas. Sample size calculation and power analysis were performed (Supplementary Figure 4). Four tracheas were excluded because of their excessively large inner diameter, which was larger than the maximum outer diameter of the cuff after inflation. Four hundred fifty-one sets of measurements were recorded (Table 2). As the ATWT correlation between the methods was poor, the resulting measurements were deemed to be inaccurate. Thus, subsequent analysis was suspended.

Correlations between CIV, TWP, OTD\%, and ITD\% as measured by ultrasound, are shown in Table 3. LOESS showed that OTD\% and ITD\% were approximately linear with respect to the CIV, despite an inflection point

Table 1 The tracheal diameter measured by precision electronic Vernier caliper and ultrasound, Mean \pm SD $(\mathrm{mm})$

\begin{tabular}{lll}
\hline Tracheal diameter & $\begin{array}{l}\text { Precision electronic } \\
\text { Vernier caliper }\end{array}$ & Ultrasound \\
\hline OTD & $26.64 \pm 2.12$ & $26.83 \pm 2.00$ \\
ITD & $21.59 \pm 1.71$ & $21.26 \pm 2.04$ \\
ATWT & $3.64 \pm 0.57$ & $3.42 \pm 0.57$ \\
\hline
\end{tabular}

OTD Outer transverse diameter, ITD Internal transverse diameter, ATWT Anterior tracheal wall thicknesses at $4 \mathrm{ml}$ (Fig. 3A-B). A strong correlation was observed between CIV and OTD\% ( $r=0.75)$, ITD\% (Four hundred fifty-one $=0.77$ ).

LOESS demonstrated that OTD\% and ITD\% were approximately linear with the TWP (Fig. 4A-B). A strong correlation was found between TWP and OTD\% $(r=0.84)$, ITD\% $(r=0.84)$.

The CIV showed a curvilinear relationship with TWP. When CIV was 0-4 mL, the TWP increased slowly. The TWP trend increased more when CIV was $4-6 \mathrm{~mL}$. When CIV was 6-10 mL, TWP increased significantly with the increase in CIV (Fig. 4C). A strong correlation was found between CIV and TWP $(r=0.75)$.

\section{Discussion}

Previous studies have shown that ultrasound can be a reliable tool for the assessment of tracheal diameter. In these studies, the tracheal outer transverse diameter (OTD) [9], internal transverse diameter (ITD) [8], and anterior tracheal wall thicknesses (ATWT) [22] were used as the principal ultrasound measurements, respectively. However, no studies have compared which diameter was measured more accurately. This experimental study assessed the accuracy of the ultrasound measurement of three separate tracheal diameters, and investigated the relationship of the percentage change of tracheal diameter, with tracheal wall pressure and cuff inflation volume.

Our results indicate that ultrasonography correlates strongly with the OTD and ITD measurements made by precision electronic Vernier caliper. And the limit of agreement for OTD was narrower. Therefore, ultrasound measurement of OTD was more accurate than measurement of ITD. The ATWT correlation between the two methods was poor, so the resulting ultrasound measurements were deemed inaccurate.

Julio [16] el at. studied the inter-rater and intra-rater reliability of ultrasound measurement of airway diameter. They showed that ultrasound measurement of ITD is both reliable and precise. Lakhal [8] el at. compared ultrasound and magnetic resonance imaging measurements of ITD. Sustic [9] et al. compared ultrasound and computed tomography measurements of OTD. In our study, there were strong correlation and agreement between the two methods when measuring the OTD and ITD. The results of our study agree closely with those of Lakhal el at. and Sustic el at.. Shih [22] el at. proposed that anterior tracheal wall thicknesses can be measured at the thyroid isthmus level with ultrasound. This result contrasted with ours. We showed that the ultrasound measurement of ATWT was inaccurate. As Shih et al. only described the results of ultrasound measurements, without applying additional verification, the inaccuracy of ATWT measurement was not apparent. Moreover, 

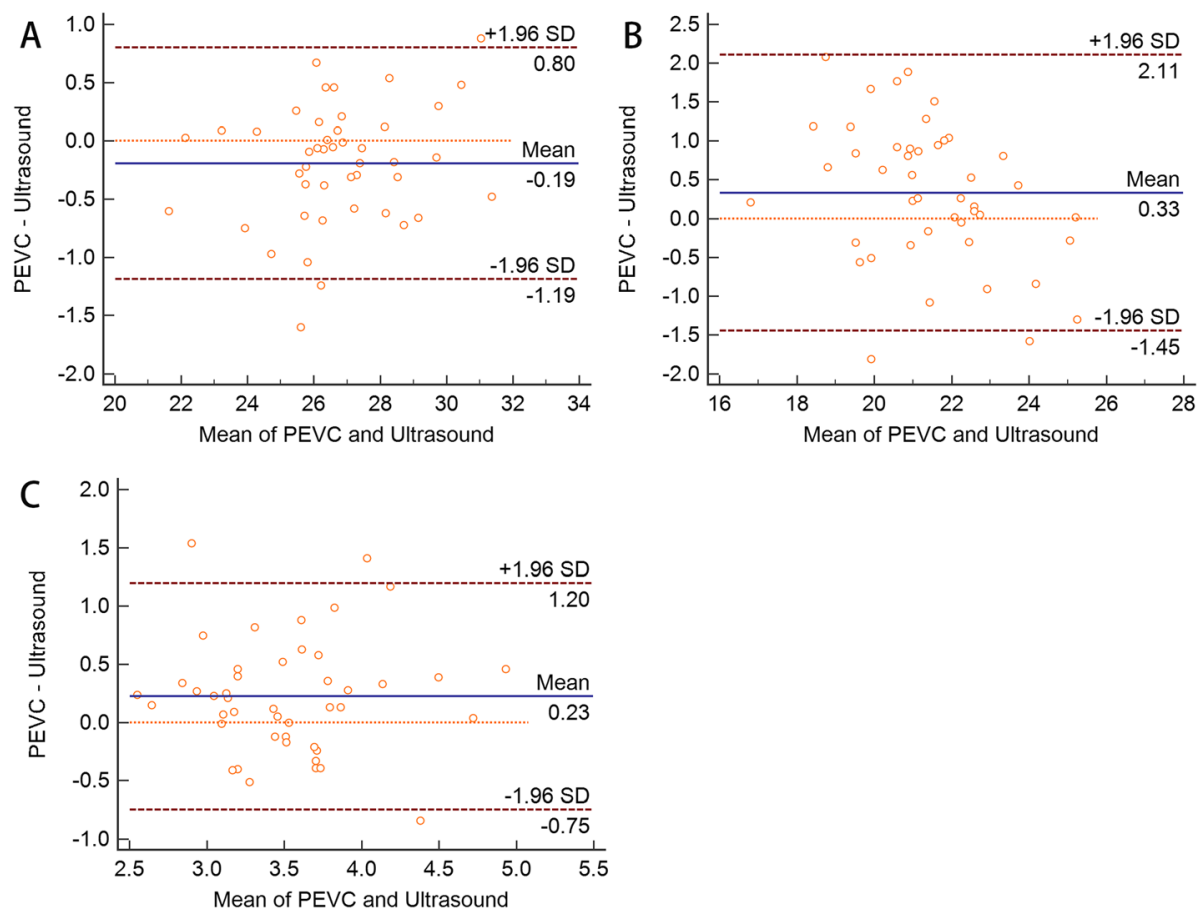

Fig. 2 Bland-Altman analysis of the precision electronic Vernier caliper (PEVC) and ultrasound measurements of tracheal outer transverse diameter $(\mathbf{A})$. Internal transverse diameter $(\mathbf{B})$. Anterior tracheal wall thicknesses $(\mathbf{C})$. The solid line indicates the bias (average difference between paired measurements) and the dotted lines indicate limits of agreement (1.96 \pm SD)

the thin anterior wall of the trachea may pose a difficulty for ultrasound measurements.

The second part of our study found that OTD\% and ITD\% measured using ultrasound correlates strongly with cuff inflation volume and tracheal wall pressure.

A study of ovine trachea explored a universally optimal cuff inflation volume [23]. The results were less than satisfactory, and their results chosen range of cuff inflation volumes $(5-7 \mathrm{ml})$ did not achieve a safe cuff pressure $\left(20-30 \mathrm{cmH}_{2} \mathrm{O}\right)$. A prospective Japanese study [24] used tracheal diameter from chest X-ray to evaluate the cuff inflation volume and compared it with an equation combining height and age. The results indicated that an equation based on tracheal diameter (Optimal cuff inflation volume $=0.71$ (tracheal diameter) -8.25 , the adjusted coefficient of determination being 0.83 ) was better

Table 2 Median (IQR) of TWP, OTD\%, and ITD\% for different cuff inflation volume

\begin{tabular}{llll}
\hline CIV $(\mathbf{m L})$ & TWP $(\mathbf{m m H g})$ & OTD\% & ITD\% \\
\hline 0 & $0(0,0)$ & $0.00(0.00,0.00)$ & $0.00(0.00,0.00)$ \\
1 & $1(1,1)$ & $0.24(-0.12,0.36)$ & $0.54(0.00,1.20)$ \\
2 & $1(1,1)$ & $0.25(0.00,0.50)$ & $0.65(0.00,1.82)$ \\
3 & $1(1,2)$ & $0.26(0.00,0.51)$ & $1.26(0.15,1.88)$ \\
4 & $2(1,3)$ & $0.37(0.13,1.03)$ & $1.50(0.81,2.25)$ \\
5 & $4(2,6)$ & $0.91(0.35,2.30)$ & $2.35(1.13,3.17)$ \\
6 & $7(3,11)$ & $1.67(0.62,3.62)$ & $3.54(2.05,5.75)$ \\
7 & $12(6,17)$ & $3.09(1.49,5.50)$ & $5.61(3.46,8.70)$ \\
8 & $17(11,23)$ & $4.34(2.65,7.25)$ & $7.74(4.65,11.92)$ \\
9 & $23(16,31)$ & $5.95(4.35,8.25)$ & $9.70(6.64,14.14)$ \\
10 & $28(20,41)$ & $7.55(5.39,9.12)$ & $11.20(8.16,14.90)$ \\
\hline
\end{tabular}

CIV Cuff inflation volume, TWP Tracheal wall pressure, OTD\% Percentage change of outer transverse diameter, ITD\% Percentage change of internal transverse diameter 
Table 3 Relationship of cuff inflation volume (CIV), tracheal wall pressure (TWP), OTD\% and ITD\%

\begin{tabular}{|c|c|c|c|c|}
\hline & \multicolumn{4}{|c|}{ Pearson Correlation coefficient, $N=451$} \\
\hline & CIV & TWP & OTD\% & ITD\% \\
\hline CIV & 1.00 & $\begin{array}{l}0.75 \\
<0.001\end{array}$ & $\begin{array}{l}0.75 \\
<0.001\end{array}$ & $\begin{array}{l}0.77 \\
<0.001\end{array}$ \\
\hline TWP & $\begin{array}{l}0.75 \\
<0.001\end{array}$ & 1.00 & $\begin{array}{l}0.84 \\
<0.001\end{array}$ & $\begin{array}{l}0.84 \\
<0.001\end{array}$ \\
\hline OTD\% & $\begin{array}{l}0.75 \\
<0.001\end{array}$ & $\begin{array}{l}0.84 \\
<0.001\end{array}$ & 1.00 & $\begin{array}{l}0.95 \\
<0.001\end{array}$ \\
\hline ITD\% & $\begin{array}{l}0.77 \\
<0.001\end{array}$ & $\begin{array}{l}0.84 \\
<0.001\end{array}$ & $\begin{array}{l}0.95 \\
<0.001\end{array}$ & 1.00 \\
\hline
\end{tabular}

CIV Cuff inflation volume, TWP Tracheal wall pressure, OTD\% Percentage change of outer transverse diameter, ITD\% Percentage change of internal transverse diameter

than the equation combining height and age (optimal cuff inflation volume $=0.11$ (height) +0.042 (age) -15.6 , the adjusted coefficient of determination being 0.44).

Some studies show that cuff inflation causes tracheal dilation, which could be observed using ultrasound on the suprasternal notch plane $[6,19]$. Therefore, ultrasound can be used instead of X-ray to measure tracheal diameter. Compared with X-ray, ultrasound has the advantage of being fast, convenient, and non-invasive, providing real-time measurements. In our study, OTD\% and ITD\% correlated strongly with the cuff inflation volume. An ultrasound-guided cuff inflation protocol should also be explored. We will investigate this in our next study.

While the cuff inflation volume is responsible for tracheal sealing, tracheal wall pressure determines potential ischemia [18]. Tracheal wall pressure is exerted by endotracheal cuff on tracheal wall. The tracheal wall pressure and the cuff pressure are distinct concepts. Some studies report tracheal wall pressure was lower than the cuff pressure [25-27]. Techniques used to measure the tracheal wall pressure included the pressure difference technique, the wall pressure membrane technique, and the microchip sensor probe technique. The wall pressure membrane technique requires perforating the trachea wall and covering it with a membrane connected to an electronic transducer. It's only suitable for in vitro studies [21]. The microchip sensor probe is known to generate artificially high pressures between cuff and trachea [28]. Both techniques are limited by the cost of acquisition and maintenance. In our study, we chose the pressure difference technique [29] for estimation of tracheal wall pressure as it is easy to use and provides relatively reliable results $[21,28]$. Its principal disadvantage is that it can only assess the overall pressure of the tracheal wall. Brimacombe [28] el at. showed the cuff will cause different tracheal wall pressure at different sites during inflation. Tracheal wall pressure has received little attention in clinical practice, which may be the reason for the lack of measurement methods. The pressure difference technique can be a method, but it is still complicated. Our results indicate a strong correlation between
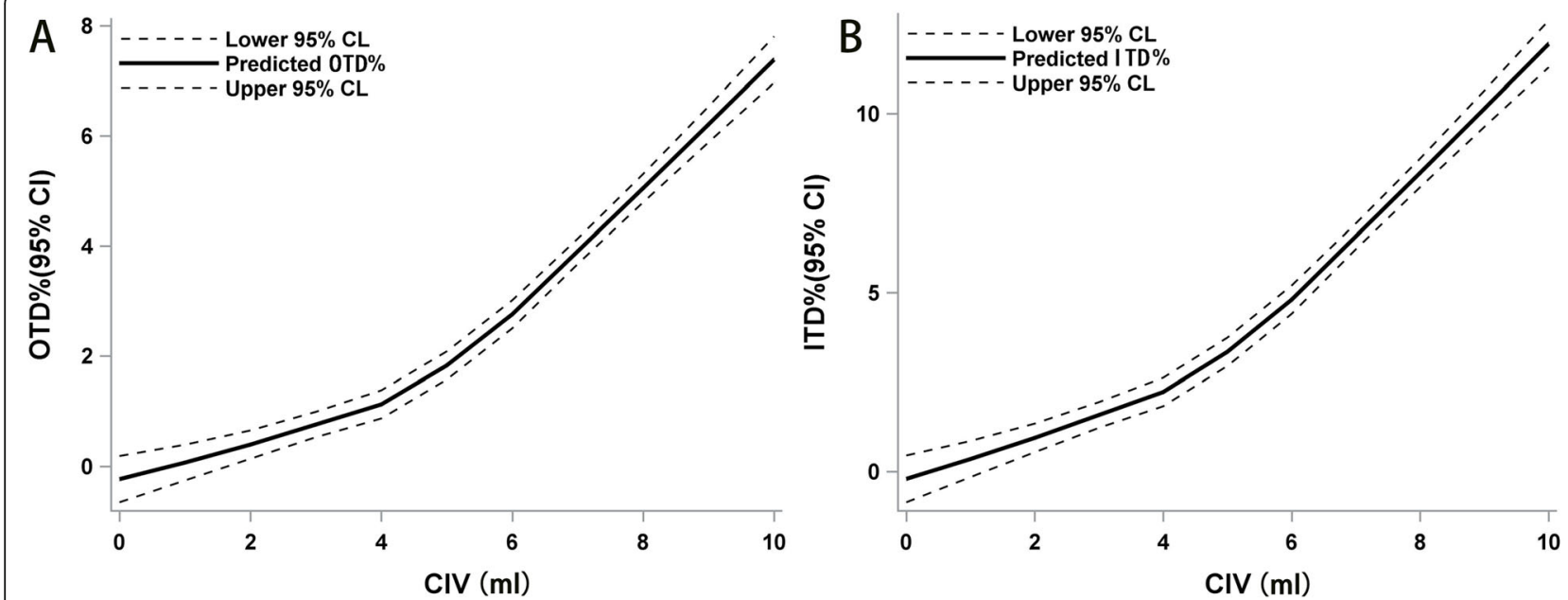

Fig. 3 The relationship of the CIV, OTD\%, and ITD\% based on LOESS. The solid line indicates the trends. The dotted lines indicate the 95\% confidence interval 

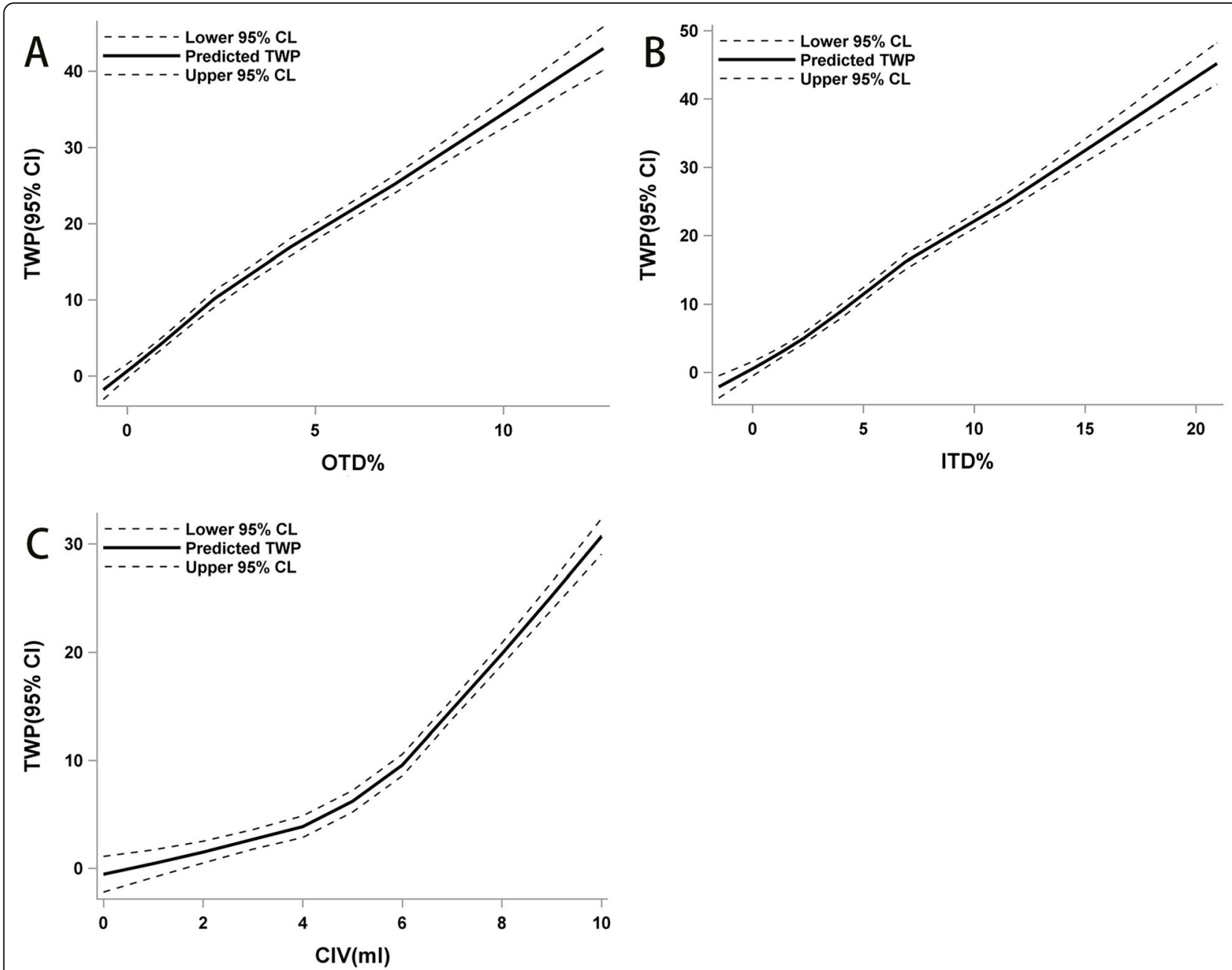

Fig. 4 The relationship of TWP, OTD\%, ITD\%, and CIV based on LOESS. The solid line indicates the trends. The dotted lines indicate the 95\% confidence interval

tracheal wall pressure and OTD\%, ITD\%. We wish to explore further whether tracheal wall pressure can be estimated using the tracheal diameter difference as measured by ultrasound.

This study has certain limitations. Firstly, we performed our study using porcine trachea in vitro rather than human trachea in vivo. Although porcine tracheal diameter is generally larger than that of humans, it is similar in structure to the human trachea. In vitro, insufficient perfusion and temperature reduction in the extracorporeal trachea may reduce the elasticity of the tissue. Secondly, we chose the $8.0 \mathrm{~mm}$ oral/nasal tracheal tube by Covidien, which is in common use within clinical settings. However, endotracheal tubes have several manufacturers, leading to different cuff inflation volume, cuff diameters, and variable composition of the materials used for the endotracheal tube. Thus, our findings may not apply to other manufacturers or sizes of endotracheal tube.

\section{Conclusions}

In conclusion, we showed that ultrasound measurements of OTD and ITD are reliable, and that the accuracy of ultrasound measurement of OTD is better than that of ITD. But the measurement of the ATWT is inaccurate. Additionally, OTD\% and ITD\%, as measured by ultrasound, correlates strongly with cuff inflation volume and tracheal wall pressure. Our study provides a basis for further development of airway ultrasound applications, such as ultrasound-guided cuff inflation protocol or using ultrasound to assess tracheal wall pressure.

\section{Abbreviation}

ETT: Endotracheal tube; TWP: Tracheal wall pressure; CIV: Cuff inflation volume; OTD: Outer transverse diameter; OTD\%: Percentage change of outer transverse diameter; ITD: Internal transverse diameter; ITD\%: Percentage change of internal transverse diameter; ATWT: Anterior tracheal wall thicknesses; ATWT\%: Percentage change of anterior tracheal wall thicknesses;

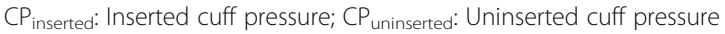




\section{Supplementary Information}

The online version contains supplementary material available at https://doi. org/10.1186/s12871-021-01398-3.

Additional file 1: Figure S1. The sample size estimated from the previous reference $[8,9]$ which their correlation coefficient is 0.882 .

Additional file 2: Figure S2. In our study, we evaluated inversely whether the sample size was sufficient. The sample size estimated with the correlation coefficient is 0.9 (Form tracheal internal transverse diameter).

Additional file 3: Figure S3. The sample size estimated from our study, with the correlation coefficient is 0.58 (Form anterior tracheal wall thicknesses)

Additional file 4: Figure S4. The sample size estimated from our study, according to the minimum correlation coefficient $(r=0.75)$ in the second part results.

\section{Acknowledgements}

Not applicable.

\section{Authors' contributions}

CSC, BC, RY and FFC conceived and designed the study; RY and FFC supervised the conduct of the study and collected data; XCZ and DY performed the literature search, and data and quality checks; CNG analyzed the data; RY drafted the manuscript; and all authors s revised the manuscript. CSC and BC take responsibility for the paper as a whole. All authors have read and approved the manuscript.

\section{Funding}

This work was partially supported by the National Key Research and Development Program of China (2016YFC1304000). The funder had no role in study design, data collection and analysis, decision to publish, or preparation of the manuscript.

\section{Availability of data and materials}

The datasets used and/or analysed during the current study are available from the corresponding author on reasonable request.

\section{Declarations}

\section{Ethics approval and consent to participate}

All animal studies were conducted under the oversight of the Institutional Animal Care and Use Committee of Wenzhou Medical University (Wenzhou, China).

\section{Consent for publication}

Not applicable.

\section{Competing interests}

The authors declare that they have no competing interests.

\section{Author details}

'Department of Ultrasonography, The second Affiliated Hospital and Yuying children's Hospital of Wenzhou Medical University, Wenzhou 325006, Zhejiang, China. 'Department of Ultrasonography, Lucheng People's Hospital of Wenzhou, Wenzhou 325006, Zhejiang, China. ${ }^{3}$ Department of Preventive Medicine, School of Public Health \& Management, Wenzhou Medical University, Wenzhou 325006, Zhejiang, China. ${ }^{4}$ Key Laboratory of Interventional Pulmonology of Zhejiang Province, The First Affiliated Hospital of Wenzhou Medical University, Wenzhou 325006, Zhejiang, China. ${ }^{5}$ Department of Pulmonary and Critical Care Medicine, Jinhua Municipal Central Hospital, Jinhua 321000, Zhejiang, China. ${ }^{6}$ Department of Pulmonary and Critical Care Medicine, The First Affiliated Hospital of Wenzhou Medical University, Wenzhou 325006, Zhejiang, China. Department of Ultrasonography, The First Affiliated Hospital of Wenzhou Medical University, Wenzhou 325006, Zhejiang, China.
Received: 24 April 2020 Accepted: 15 June 2021

Published online: 24 June 2021

\section{References}

1. You-Ten KE, Siddiqui N, Teoh WH, Kristensen MS. Point-of-care ultrasound (POCUS) of the upper airway. Can J Anaesth. 2018;65:473-84.

2. Mandeep S, Ki Jinn C, Chan WWS, Wong DT, Prasad GA, Eugene Y. Use of sonography for airway assessment: an observational study. J Ultrasound Med Official J Am Inst Ultrasound Med. 2010;29:79.

3. Werner SL, Smith CE, Goldstein JR, Jones RA, Cydulka RK. Pilot study to evaluate the accuracy of ultrasonography in confirming endotracheal tube placement. Ann Emerg Med. 2007:49:75-80

4. Bunyamin $M$, Hüseyin $S$, Ahmet $K$, Rüveyda Irem D, Muhammet GZ, Burhanettin $U$, et al. Use of sonography for rapid identification of esophageal and tracheal intubations in adult patients. J Ultrasound Med. 2011;30:671-6.

5. Hoffmann B, Gullett JP, Hill HF, Fuller D, Westergaard MC, Hosek WT, et al. Bedside ultrasound of the neck confirms endotracheal tube position in emergency intubations. Ultraschall Med. 2014;35:451-8.

6. Giabicani M, Compere V, Fourdrinier V, Dureuil B. Is sickle cell disease a possible risk factor for peripheral neuropathy after popliteal sciatic nerve block? Br J Anaesth. 2013;111:508-10.

7. Dong F, Zhu C, Xu H, Wang J, Zhu Y, Fan Q, et al. Measuring endotrachea tube depth by bedside ultrasound in adult patients in an intensive care unit: a pilot study. Ultrasound Med Biol. 2017;43:1163-70.

8. Lakhal K, Delplace X, Cottier JP, Tranquart F, Sauvagnac X, Mercier C, et al. The feasibility of ultrasound to assess subglottic diameter. Anesth Analg. 2007;104:611-4

9. Sustic A, Miletic D, Protic A, Ivancic A, Cicvaric T. Can ultrasound be useful for predicting the size of a left double-lumen bronchial tube? Tracheal width as measured by ultrasonography versus computed tomography. Clin Anesth. 2008;20:247-52.

10. Eom K, Moon K, Seong Y, Oh T, Yi S, Lee K, et al. Ultrasonographic evaluation of tracheal collapse in dogs. J Vet Sci. 2008:9:401-5.

11. Sutherasan $Y$, Theerawit $P$, Hongphanut $T$, Kiatboonsri $C$, Kiatboonsri $S$. Predicting laryngeal edema in intubated patients by portable intensive care unit ultrasound. J Crit Care. 2013;28:675-80.

12. Ding LW, Wang HC, Wu HD, Chang CJ, Yang PC. Laryngeal ultrasound: a useful method in predicting post-extubation stridor. A pilot study. Eur Respir J. 2006;27:384-9.

13. Stuntz R, Kochert E, Kehrl T, Schrading W. The effect of sonologist experience on the ability to determine endotracheal tube location using transtracheal ultrasound. Am J Emerg Med. 2014:32:267-9.

14. Gottlieb M, Bailitz JM, Christian E, Russell FM, Ehrman RR, Khishfe B, et al. Accuracy of a novel ultrasound technique for confirmation of endotracheal intubation by expert and novice emergency physicians. West J Emerg Med. 2014;15:834-9.

15. Chou H-C, Tseng W-P, Wang C-H, Ma MH-M, Wang H-P, Huang P-C, et al. Tracheal rapid ultrasound exam (T.R.U.E.) for confirming endotracheal tube placement during emergency intubation. Resuscitation. 2011:82:1279-84.

16. Gomez-Tamayo JC, Puerta-Guarin Jl, Rojas-Camejo CM, Caicedo JP, Calvache JA. Inter-rater and intra-rater reliability of the airway diameter measured by sonography. J Ultrasound. 2018;21:35-40.

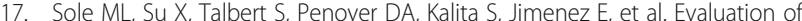
an intervention to maintain endotracheal tube cuff pressure within therapeutic range. Am J Crit Care. 2011:20:109-17.

18. Seegobin RD, Hasselt GLV. Endotracheal cuff pressure and tracheal mucosal blood flow: endoscopic study of effects of four large volume cuffs. Br Med J. 1984;288:965-8

19. Ramsingh D, Frank $E$, Haughton $R$, Schilling J, Gimenez KM, Banh E, et al. Auscultation versus point-of-care ultrasound to determine endotracheal versus bronchial intubation: a diagnostic accuracy study. Anesthesiology. 2016:124:1012-20

20. Ding XD, Chen $H X$, Xiao HQ, Wang W, Zhang GB. Efficiency of ultrasound and water capsule-guided local injection of botulinum toxin type a treatment on patients with facial spasm. Eur Rev Med Pharmacol Sci. 2015 19:1837-41.

21. Horisberger T, Gerber S, Bernet V, Weiss M. Measurement of tracheal wall pressure: a comparison of three different in vitro techniques. Anaesthesia. 2008;63:418-22. 
22. Shih JY, Lee LN, Wu HD, Yu CJ, Wang HC, Chang YL, et al. Sonographic imaging of the trachea. J Ultrasound Med. 1997;16:783-90.

23. Carhart E, Stuck LH, Salzman JG. Achieving a safe endotracheal tube cuff pressure in the prehospital setting: is it time to revise the standard cuff inflation practice? Prehosp Emerg Care. 2016;20:273-7.

24. Shibasaki M, Nakajima Y, Shime N, Sawa T, Sessler DI. Prediction of optimal endotracheal tube cuff volume from tracheal diameter and from patient height and age: a prospective cohort trial. J Anesth. 2012;26:536-40

25. Black AMS, Seegobin RD. Pressures on endotracheal tube cuffs. Anaesthesia. 1981;36:1.

26. Bernet V, Dullenkopf A, Cannizzaro V, Stutz K, Weiss M. An in vitro study of the compliance of paediatric tracheal tube cuffs and tracheal wall pressure. Anaesthesia. 2006;61:978-83.

27. Dobrin P, Canfield T. Cuffed endotracheal tubes: mucosal pressures and tracheal wall blood flow. Am J Surg. 1977:133:562-8.

28. Brimacombe J, Keller C, Giampalmo M, HJ S, Berry A. Direct Measurement of Mucosal Pressures Exerted by Cuff and Non-Cuff Portions of Tracheal Tubes with Different Cuff Volumes and Head and Neck Positions. Surv Anesthesiol. 2000;82(5):708.

29. Mackenzie CF, Klose S, BROWNE DRG. A study of inflatable cuffs on endotracheal tubes: pressures exerted on the trachea. BJA Br J Anaesth. 1976:48:105-10

\section{Publisher's Note}

Springer Nature remains neutral with regard to jurisdictional claims in published maps and institutional affiliations.

Ready to submit your research? Choose BMC and benefit from:

- fast, convenient online submission

- thorough peer review by experienced researchers in your field

- rapid publication on acceptance

- support for research data, including large and complex data types

- gold Open Access which fosters wider collaboration and increased citations

- maximum visibility for your research: over $100 \mathrm{M}$ website views per year

At BMC, research is always in progress.

Learn more biomedcentral.com/submissions 